\title{
Fluorescence in situ hybridization analysis of hobo, $m d g 1$ and Dm412 transposable elements reveals genomic instability following the Drosophila melanogaster genome sequencing
}

\author{
LP Zakharenko ${ }^{1,2,3}$, LV Kovalenko ${ }^{1}$ and S Mai ${ }^{3}$ \\ ${ }^{1}$ Siberian Department, Institute of Cytology and Genetics of the Russian Academy of Sciences, Novosibirsk, Russia; ${ }^{2}$ Department of \\ Cytology and Genetics, Novosibirsk State University, Novosibirsk, Russia and ${ }^{3}$ Manitoba Institute of Cell Biology, The University \\ of Manitoba, CancerCare, Manitoba, Winnipeg, Canada
}

\begin{abstract}
The genome of Drosophila melanogaster strain y cn bw sp has been sequenced and the transposable elements insertion sites have been determined. We hybridized fluorescence-labeled probes directed to the hobo transposon, Dm412 and mdg1 retrotransposons to polytene chromosomes and compared the observed sites to those published in the annotated genome sequence. We observed an almost twofold increase in the number of hobo hybridization sites (46 found as compared to 24 annotated sites). There was no
\end{abstract}

Keywords: strain y cn bw sp; hobo; P-element; mdg1; Dm412 evidence that the hobo transposition rate is slowing over the 10 -year period. The patterns of Dm412 and $m d g 1$ sites have changed less dramatically since the time of genome sequencing. Three novel Dm412 hybridization sites were detected while 4 out of 30 annotated sites were missing. Only one additional $m d g 1$ site was found, while 1 out of 29 annotated sites has been lost.

Heredity (2007) 99, 525-530; doi:10.1038/sj.hdy.6801029; published online 11 July 2007

\section{Introduction}

The presence of active transposable elements (TEs) in the genome is an important factor responsible for genetic instability. As much as $3.8 \%$ of euchromatin (Kaminker et al., 2002) and up to $22 \%$ of total DNA (Kapitonov and Jurka, 2003) in Drosophila melanogaster is composed of TEs. The spontaneous rate of TE transposition in Drosophila is about $10^{-3}-10^{-5}$ per site per generation depending on the genetic background and environmental conditions (Harada et al., 1990; Nuzhdin et al., 1996; Vieira and Biemont, 1997; Lampe et al., 1998; Maisonhaute et al., 2007).

$P$ and hobo transposons invaded the D. melanogaster genome not earlier than 100 years ago (Blackman et al., 1987; Bazin et al., 1999). According to the hypothesis of Rouzic and Capy (2005), the transposition rate can vary by several orders of magnitude during the colonization process. They propose that the rate is high during the first generations following the horizontal transfer, but then decreases until an equilibrium value is reached corresponding to the known measured transposition rates (Rouzic and Capy, 2005).

TEs are now widely used to generate genome-wide mutant collections. $P$ elements have been the vehicle most often employed to disrupt Drosophila genes because

Correspondence: Dr LP Zakharenko, Siberian Department, Institute of Cytology and Genetics, Lavrent'eva 10, Novosibirsk 630090, Russia.

E-mail: zakharlp@bionet.nsc.ru

Received 26 November 2006; revised 6 June 2007; accepted 9 June 2007; published online 11 July 2007 they transpose at the highest rates (Bellen et al., 2004). The minimal rate of transposition of another newcomer, hobo, was evaluated as $1.75 \times 10^{-3}$ per site per generation in the strains studied by Harada et al. (1990). However, Aulard et al. (2004) show that a hobo copy artificially introduced into the fly genome previously free of hobo insertions transposes at the rate of $0.19-0.45$ per site per generation.

The isogenic strain $y c n$ bw sp of D. melanogaster is used by many researchers as the reference strain, in large part, because a complete genome sequence is available (Adams et al., 2000; Myers et al., 2000). This strain carries full-sized copies of hobo, Dm412 and mdg1 elements capable of inducing transpositions. It is, therefore, tempting to study the stability of TE patterns both for the newcomer hobo transposon and the ancient Dm412 and $m d g 1$ retrotransposons in this strain, and determine transposition rates for those that are active. This may be of interest for understanding genome instability from an evolutionary perspective.

We found that the number of hobo hybridization sites on polytene chromosomes of the $y$ cn bw sp genome is higher than that annotated in the database (http:// flybase.bio.indiana.edu). In contrast, Dm412 and $m d g 1$ hybridization sites largely coincided with those annotated in silico.

\section{Materials and methods}

Fluorescence in situ hybridization (FISH) experiments were performed with $y[1]$ oc[R3.2]; cn[1] bw[1] sp[1]; LysC[1] MstProx[1] GstD5[1] Rh6[1] strain (hereafter 
referred to as y $c n$ bw $s p$ ) obtained from Bloomington Drosophila Stock Center (USA) in summer 2004 and summer 2005. Flies of the isogenic strain y $c n$ bw $s p$ of D. melanogaster are suitable for the analysis of mobility because this stock has been made isogenic and labeled with recessive phenotypic markers. Thus, all heterogeneity should be due to novel instability as opposed to drift of preexisting polymorphism or contamination.

The cytological localization of hobo element, Dm412 and $m d g 1$ in the nearly completely sequenced genome was performed with the use of FlyBase Insertions query results (http://flybase.net/transposons). Information concerning hobo (or H-element), Dm412 (or 412) and mdg1 (or mdg1) in sequenced genome is designated as H\{\}\# (2003), hobo $\{2005\} \#, 412\{\} \#$ and mdg1\{\}\#, respectively in the database.

FISH was performed on the squashed preparations of larval salivary glands. We used 4-5 squashed preparations for every type of probe. Slides were heated at $60^{\circ} \mathrm{C}$ for $1 \mathrm{~h}$ and denatured in $0.07 \mathrm{M} \mathrm{NaOH}$ for $3 \mathrm{~min}$. The completely cloned copies of hobo transposon (provided by J Lim), Dm 412 and $m d g 1$ (provided by N Fedorova) were used as a probe. The probe was labeled by nick translation with biotinylated dUTP (Medigen, Novosibirsk, Russia), Cy3-UTP (Roche oligolabeling kit) or digoxigenin-dUTP (Molecular Probes, Invitrogen, Eugene, OR, USA). The in situ hybridization solution was as follows: $50 \%$ formamide, $10 \%$ dextransulfate, $4 \times$ SSC, $1 \times$ Denhardt solution, $0.1 \mathrm{M}$ phosphate buffer ( $\mathrm{pH}$ 7.6) and 10-20 ng labeled DNA per slide. After hybridization, the squashed preparations were washed with $2 \times$ SSC three times for $5 \mathrm{~min}$. The detection of biotin was performed with avidin-fluorescein isothiocyanate (avidin-FITC), digoxigenin was detected by antidigoxigenin-Cy3 (Molecular Probes). Vectashield with 4',6-diamidino-2-phenylindole (DAPI; Vector Laboratories, Inc., Burlingame, CA, USA) was used as an antifading solution.

Following the FITC-DNA hybridization and FITC/ Cy3-DNA hybridization, the preparations were examined with an Axioskop-2 Plus microscope equipped with a black-and-white CCD VC44 camera (PCO). The images were processed with the ISIS program (METSYSTEMS $\mathrm{GmbH})$. The result of Cy3-labeled Dm412 DNA hybridization was analyzed on Zeiss Axiophot2 microscope equipped by Zeiss Axio Cam HRm. The images were processed with the AxioVision version 3.1.

\section{Results}

We compared the in silico localization sites of hobo, Dm412 and $m d g 1$ TEs in the $y c n$ bw sp strain of D. melanogaster to our FISH data. Since the correspondence between genome sequence and the cytological map is known (with a polytene chromosome bandwidth accuracy) in silico data can be used to localize a mobile element on the cytological map, to allow this comparison.

By 2003, as many as 24 hobo sequences had been annotated in the database for $y c n$ bw $s p$ strain. One of these annotated sequences represents the full-sized copy (2959 bp) encoding the active transposase (accession number M69216), while the other hobo copies carry deletions in the central part (http://flybase/). A 1406bp-long variant is a predominantly truncated version with high homology to a full-sized hobo. According to the database, the hobo element is distributed non-uniformly on the chromosomes. Thus, the $\mathrm{X}$ chromosome contains five copies while $2 \mathrm{~L}, 2 \mathrm{R}, 3 \mathrm{~L}$, and $3 \mathrm{R}$ carry $10,2,1$ and 6 hobo hybridization sites, respectively.

In comparison to the database localizations, the number of FISH hobo sites is almost twofold higher (46 sites against 24); X chromosome has 7; 2L, 13; 2R, 3; 3L, 6; 3R, 17 hobo hybridization sites (Figure 1 and Table 1). For example, out of five annotated X-linked sites, two sites from chromocenter region were lost, while we were able to determine four novel sites in the regions $12 \mathrm{EF}$, 13EF, 16A and 18EF (Figure 2). Some sites from 3R chromosome are polymorphic (Table 1).

The same FISH experiments performed a year later (in 2005) revealed the additional hobo pattern changes in $X$ and 3R chromosomes, namely, the loss of 18EF site and generation of a de novo X-linked site in the 16EF region. Novel 9A and 14C hobo sites were observed in a single slide out of five analyzed (Figure 3 and Table 1). The loss of four annotated sites and generation of one new site occurred in 3R chromosome (Figure 4 and Table 1). In addition, we observed the case of hobo localization polymorphism in 93 region of 3R chromosome (Figure 5). Gray arrows show hobo positions in different homologs on the different sides of $m d g 1$ in the 93rd region. No hobopattern changes were observed in the other chromosomes in 2005 experiments as compared to 2004 (Table 1). In 2005, the database was supplemented with 39 additional defective hobo elements that had less homology with full-sized hobo and were much shorter than those annotated in 2003 (most of them being 100-300 bp derivatives). However, those additional potential hobo hybridization sites on $\mathrm{X}$ chromosome were annotated to the regions 11B, 20B, 20D1 and 20D2, whereas in our experiments, hybridization was observed in the other

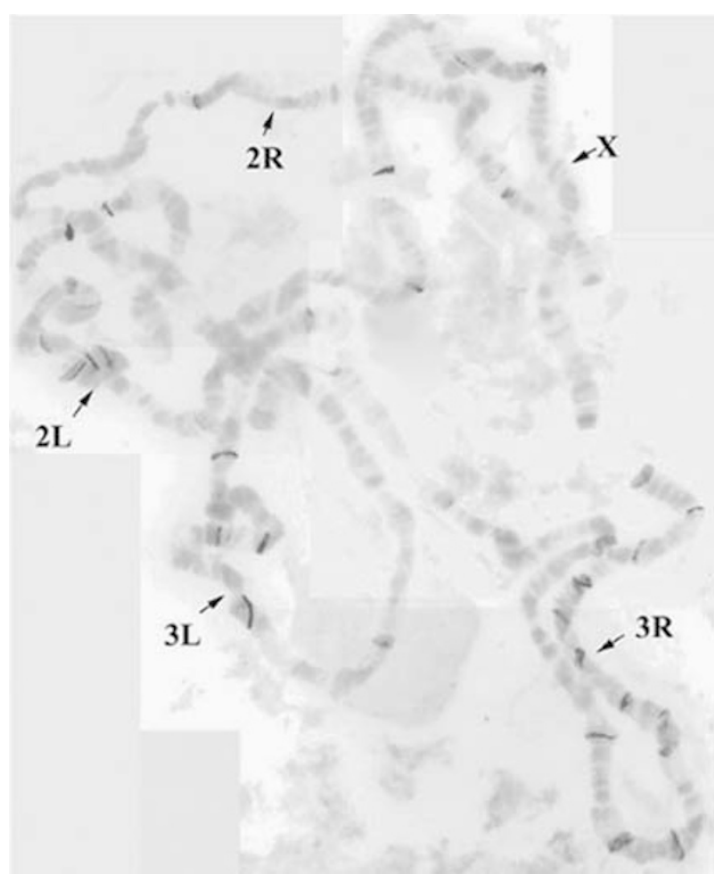

Figure 1 Fluorescence in situ hybridization (2004) of fluorescein isothiocyanate-labeled hobo DNA on the salivary gland polytene chromosomes from $y c n$ bw $s p$ strain. Arrows show chromosome arms. 
Table 1 Distribution of hobo transposable element on y $c n$ bw $s p$ polytene chromosomes according to FISH (2004 and 2005) and in silico data

\begin{tabular}{lll}
\hline In silico & FISH (2004) & FISH (2005) \\
\hline X & & \\
7E1, 9D3, 19C5, & 7E, 9D, 12EF, 13EF, & 7E, 9A(1/5), 9D, 12EF, \\
19E5, 20B1 & 16A, 18EF, 20 & 13EF, 14C(1/5), 16A, \\
& & 16F, 20
\end{tabular}

\section{$2 \mathrm{~L}$}

34A2 (two sites),

35D3 (two sites),

35D4 (two sites),

$36 \mathrm{C} 2,36 \mathrm{D} 2,36 \mathrm{E} 2$,

$38 \mathrm{C} 2$

$26 \mathrm{AB}, 26 \mathrm{~F}, 28 \mathrm{CB}$, 29CD, 30BC, 31A $33 \mathrm{AB}, 33 \mathrm{D}, 34 \mathrm{CD}$ (two sites), 35D

(two sites), 38C

$26 \mathrm{AB}, 26 \mathrm{~F}, 28 \mathrm{CB}$, 29CD, 30BC, 31A $33 \mathrm{AB}, 33 \mathrm{D}, 34 \mathrm{CD}$ (two sites), 35D

(two sites), 38C

2R $45 \mathrm{E} 1,45 \mathrm{E} 1$

$50 \mathrm{~A}, 55 \mathrm{AB}, 59 \mathrm{AB}$

$50 \mathrm{~A}, 55 \mathrm{AB}, 59 \mathrm{AB}$

$3 \mathrm{~L}$

67A1

61E, 67A, 67DE, 70E, 61E, 67A, 67DE, 70E， $71 \mathrm{~A}, 74 \mathrm{~A}$ $71 \mathrm{~A}, 74 \mathrm{~A}$

$3 R$ 84B5, 84D11, 90D1 95E1, 99B9, 99D5

$84 \mathrm{~B}, 84 \mathrm{D}(2 / 3), 86 \mathrm{~A}$, $86 \mathrm{D}(3 / 4), 89 \mathrm{~A}, 90 \mathrm{D}$, 92A, 93E, 94A(2/4), $95 \mathrm{~A}(2 / 4), 96 \mathrm{~B}(2 / 4)$ 96F, 97A, 97C(1/4), $98 \mathrm{~B}, 99 \mathrm{~F}(2 / 4), 100 \mathrm{~F}$

Total 24

46

45

Abbreviations: FISH, fluorescence in situ hybridization; y cn bw sp, y[1] oc[R3.2]; cn[1] bw[1] sp[1]; LysC[1] MstProx[1] GstD5[1] Rh6[1]. In case hybridization patterns are polymorphic, ratio of the slides with hybridization to the total number of slides analyzed is given in parentheses. Not less than three genomes per slide were analyzed.

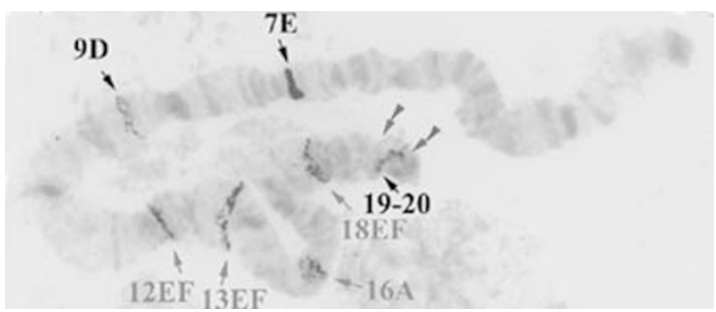

Figure 2 Fluorescence in situ hybridization (2004) of fluorescein isothiocyanate-labeled hobo DNA on the salivary gland X chromosome from y cn bw sp strain. Black arrows show annotated positions; gray arrows, new sites; double arrows, lost sites.

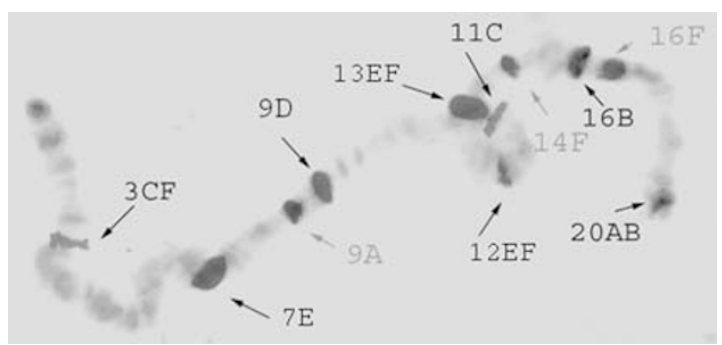

Figure 3 Fluorescence in situ hybridization (FISH; 2005) of fluorescein isothiocyanate-labeled hobo DNA (black arrows) and Cy3-labeled $m d g 1$ DNA (3CF and 11C) on the X chromosome from $y$ cn bw sp strain. Gray arrow shows the position of the new hobo sites in comparison with FISH (2004).

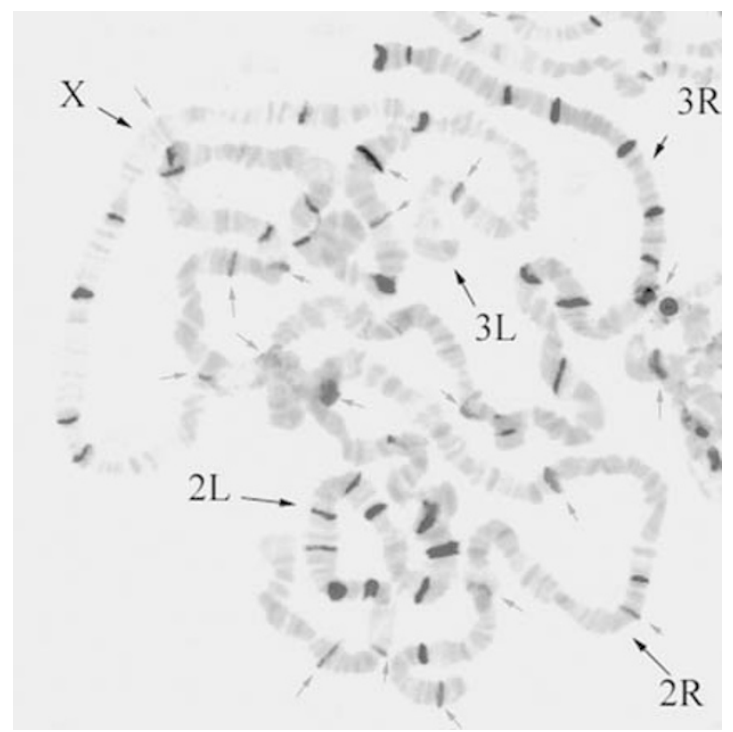

Figure 4 Fluorescence in situ hybridization (2005) of fluorescein isothiocyanate-labeled hobo DNA and Cy3-labeled mdg1 DNA on the salivary gland polytene chromosomes from y $c n b w s p$ strain. Black arrows show chromosome arms, gray arrows show mdg1 hybridization sites.

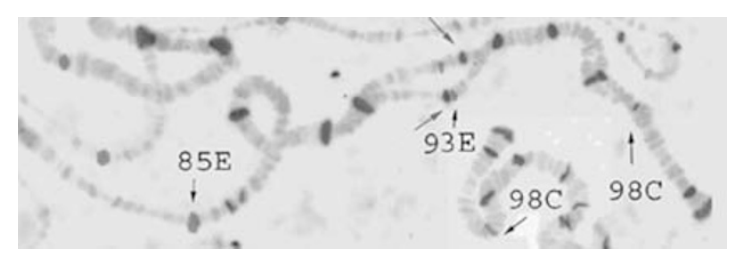

Figure 5 Fluorescence in situ hybridization (2005) of fluorescein isothiocyanate-labeled hobo DNA and Cy3-labeled $m d g 1$ DNA on 3R chromosome from $y c n b w s p$ strain. Arrows show positions of $m d g 1$ hybridization sites (85E, 93F, 98C). The polymorphic hobo hybridization pattern is shown in 93 regions.

locations indicated above. Only three out of six annotated hobo sites (in regions 84B, 84D and 90D) are present in the 3R chromosome in our FISH data. Fourteen novel hobo hybridization sites also do not colocalize with those annotated additionally in silico in 2005 (Tables 1 and 2). In addition, those six sequences annotated in silico in 2005 represent short hobo derivatives with a low homology, thus, probably, being below FISH sensitivity (Table 2). We, therefore, believe that the novel hobo hybridization sites detected by FISH most likely appeared as a result of de novo transpositions.

We were able to detect $20 m d g 1$ hybridization sites as compared to 29 annotated sites (Figure 4 and Table 3). It is noteworthy that $m d g 1$ sites are found more often then hobo in the same band or neighboring bands of the polytene chromosome. In FISH experiments, this results in a single visible hybridization site instead of two sites actually present. In such twin cases, the loss of one copy might go unnoticed. We observed only one additional mdg1 site compared to the in silico data, 11C (Figure 3), while one site from the $27 \mathrm{C} 7$ region was lost (Table 3).

FISH analysis of Dm412 retrotransposon distribution also yielded a better correspondence to in silico data (Figures 6a and b) than the hobo case. Out of 30 annotated insertions of Dm412, four sites (1E1, 41E3, 41E5 and 
Table 2 Size and cytological localization of hobo sequences in 3R chromosome of $y \mathrm{cn}$ bw sp strain according to in silico data

\begin{tabular}{lrc}
\hline $\begin{array}{l}\text { Annotated in silico } \\
H \text { (2003), hobo (2005) }\end{array}$ & hobo size $(b p)$ & $\begin{array}{c}\text { Cytological map } \\
\text { position (in silico) }\end{array}$ \\
\hline hobo\{\}2835 & 275 & $86 \mathrm{C} 10$ \\
hobo\{\}4793 & 433 & $87 \mathrm{C} 1$ \\
$\mathrm{H}\{\} 1380$ & 1406 & $90 \mathrm{D} 1$ \\
hobo\{\}5686 & 98 & $91 \mathrm{~F} 2$ \\
hobo\{\}4815 & 552 & $92 \mathrm{D} 1$ \\
$\mathrm{H}\{\} 1414$ & 1406 & $95 \mathrm{E} 1$ \\
hobo\{\}2368 & 35 & $96 \mathrm{D} 6$ \\
hobo\{\}2918 & 80 & $98 \mathrm{~A} 10$ \\
$\mathrm{H}\{\} 1453$ & 1406 & $99 \mathrm{~B} 9$ \\
$\mathrm{H}\{\} 1456$ & 1406 & $99 \mathrm{D} 5$
\end{tabular}

Abbreviation: y cn bw sp, y[1] oc[R3.2]; cn[1] bw[1] sp[1]; LysC[1] MstProx[1] GstD5[1] Rh6[1].

Table 3 Distribution of $m d g 1$ transposable element on the $y$ cn bw sp polytene chromosomes according to FISH and in silico data

\begin{tabular}{ll}
\hline In silico & FISH (2005) \\
\hline $\begin{array}{l}\text { X-chromosome } \\
\text { 3C1-3C2, 3F1, 16B4, 20A1, 20B1 }\end{array}$ & 3CF, 11C, 16B, 20AB \\
2L & 23D, 25A, 40F \\
23D6, 25A1, 27C7, 40F7 & \\
2R & 41DE, 47B, 51D, 56F, 60D \\
41C5, 41D2, 41D3, 41E1, 47B1, 47B4, & \\
51D8, 56F2, 60D1 & \\
3L & 61E, 63A, 74F/75A, 75F, \\
61E1, 63A2, 74F3, 75A1, 75F8, 80C1, & \\
80D5, 80F9 & 85E, 93F, 98C \\
3R & 85E11, 93F9, 98C2
\end{tabular}

Abbreviations: FISH, fluorescence in situ hybridization; y cn bw sp, y[1] oc[R3.2]; cn[1] bw[1] sp[1]; LysC[1] MstProx[1] GstD5[1] Rh6[1]. Closely located $m d g 1$ insertions that cannot be resolved by FISH are marked in italics.

77D4) were lost, whereas three novel sites (21CD, 39CD and 59B) were detected (Figures $6 \mathrm{a}$ and b). In region 60 of $2 \mathrm{R}$ chromosome, we sometimes observe only three Dm412 sites instead of four annotated, which may be explained by a close localization of 60C and 60D Dm412 sites, poorly resolvable by FISH. No changes in Dm412 pattern were detected by FISH performed in 2005 as compared to the FISH results obtained a year earlier (not shown), thus testifying a lack of its transposition activity over this period.

\section{Discussion}

We observed clear differences in the distribution of the three TEs between the in silico records and our FISH data for the $y c n$ bw $s p$ strain. This difference is most prominent for the hobo element, apparently indicating a high transposition activity. The frequency of hobo insertions is higher than that of excisions, as confirmed by the numbers of hybridization sites appearing de novo and disappearing (Table 1).

Assuming that the DNA used for sequencing the complete D. melanogaster genome was isolated 10-15 years ago (Smoller et al., 1991; Tamkun et al., 1992; Shaffer et al., 1994; Adams et al., 2000) and that the fly life span under laboratory conditions is approximately 1 month, at least 100-150 generations have passed over that period. During that time, 10 hobo sites (out of the 24 annotated) have been lost, while 36 new sites have been generated (Table 1).

Since we are unable to follow all intermediate events of appearance/disappearance of sites, the minimal rate of hobo element transposition is $3 \times 10^{-3}$ per site per generation for excision $(10 / 24 \times 150)$ and $10 \times 10^{-3}$ per site per generation for insertion $(36 / 24 \times 150)$. The transposition frequency calculated for both excisions and insertions is $13 \times 10^{-3}$ per site per generation (46/ $24 \times 150)$. We cannot distinguish closely located hybridization sites by FISH, which means that we have probably underestimated the hobo transposition rate.

Assuming that all hobo elements transpose at the same rate, the data suggest that at least 1 hobo copy out of nearly 50 found in $y c n$ bw sp strain will transpose in every second generation; this rate translates into six transpositions per genome per year. Over the period of 12 months (2004-2005), we have observed 4 novel hobo sites out of 46 , while 5 sites were lost. The average frequency of transposition over this period is $16 \times 10^{-3}$ $(9 / 46 \times 12)$ or $12 \times 10^{-3}(6.4 / 46 \times 12)$ taking into account the polymorphic nature of some sites. Comparing the average transposition rate over 1-year and the 10-year period, we can conclude that hobo transposition rate did not diminish with time.

Dm412 and mdg1 transposition rates are an order of magnitude lower than that of hobo element in y cn bw $s p$ strain, being about $2 \times 10^{-3}(7 / 30 \times 150)$ per site per generation for $D m 412$ and $5 \times 10^{-4}(2 / 29 \times 150)$ per site per generation for $m d g 1$.

D. melanogaster has nearly 100 different TEs in its genome (Kaminker et al., 2002). Assuming that every type of transposable element has an average of 10 copies per genome (Rouzic and Capy, 2005), and that the average transposition rate is in the range $10^{-3}-10^{-5}$ (Nuzhdin et al., 1996; Vieira and Biemont, 1997), we can conclude that the total rate of $\mathrm{TE}$ transposition will be close to the transposition rate of hobo obtained in our study.

In comparing the transposition rates of transposons (hobo) and retrotransposons (Dm412 and mdg1), we should also consider the difference in the mechanism of transposition. Retroelements transpose via an RNA intermediate by a 'copy and paste' mechanism while transposons transpose by 'cut and paste' process in the DNA form. The 'copy and paste' process might be more time-consuming than the 'cut and paste', since it involves the reverse transcription step in cytoplasm, while the 'cut and paste' mechanism is completely localized to the nucleus. Possibly, retroelements are less active than the tranposons simply due to the different mechanism of transposition.

Isogenization does not rescue the Drosophila strains from instability. Moreover, during the process of isogenization the genome instability might be increased. One cannot rule out the possibility of hobo-mediated hybrid dysgenesis, when crossing the flies of hobocarrying strains with the individuals free of this element, thus inducing the genome instability (Blackman et al., 1987; Bazin et al., 1999). Strain y cn bw sp of 

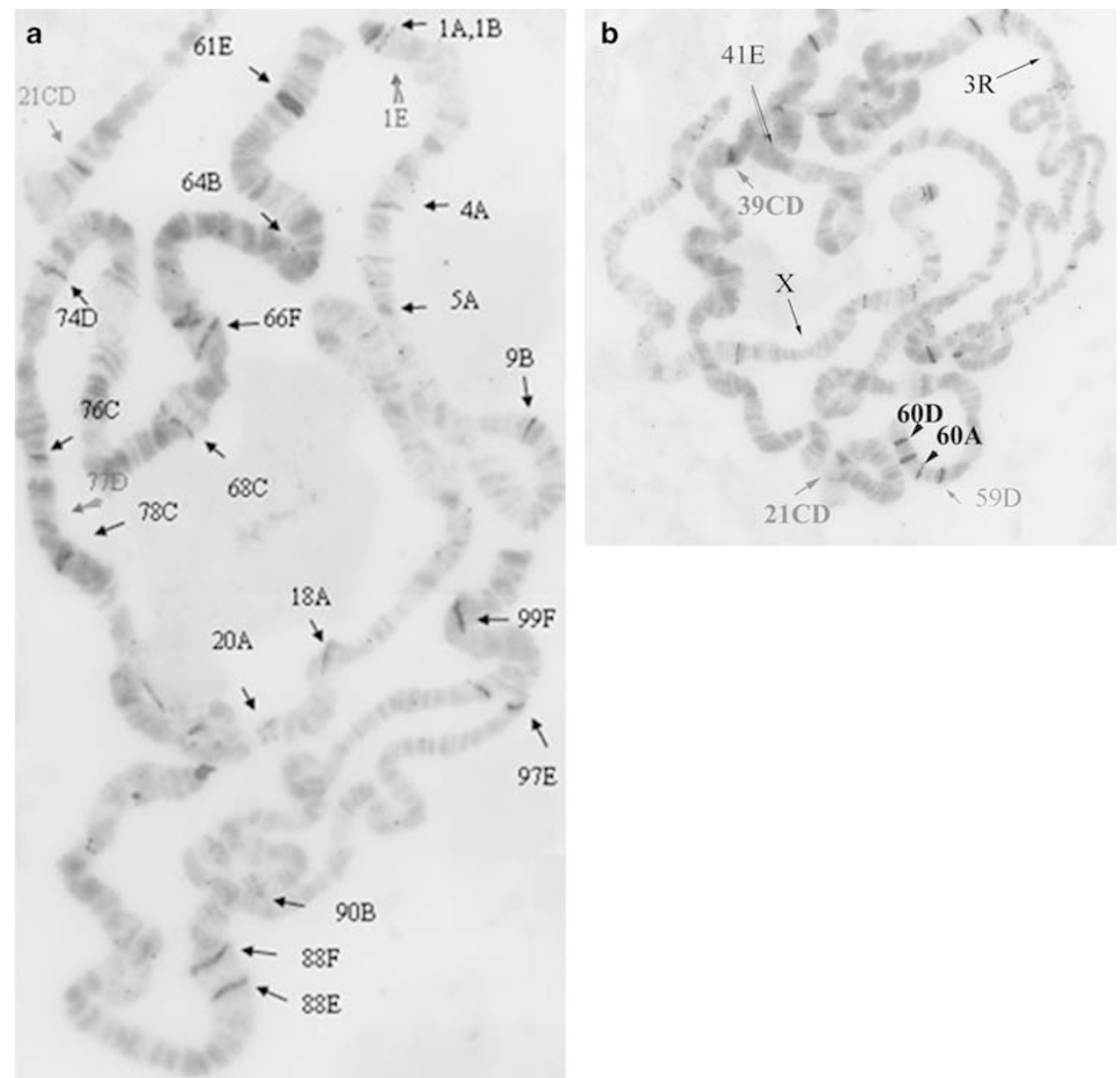

Figure 6 Fluorescence in situ hybridization (2004) of Cy3-labeled Dm412 DNA on the salivary gland X chromosome and third chromosome (a) and $\mathrm{X}$ and second chromosome (b) from y $c n$ bw sp strain (black arrows-annotated positions; gray arrows-new sites; double arrowslost sites). Two closely located lost sites are annotated to region 41 (41E3 and 41E5).

D. melanogaster was isogenized before DNA separation for sequencing, which may account for the high hobo instability.

\section{Acknowledgements}

This work was partially funded by the Russian Fund of Fundamental Research Nos. 05-04-48838, 04-140-48116 and by the Canadian Institutes of Health Research (CIHR) Strategic Training Program 'Innovative Technologies in Multidisciplinary Health Research'. We thank both referees for valuable comments. We thank I Zakharov for reading the manuscript and I Ivanoshuk and M Perepelkina for technical assistance.

\section{References}

Adams MD, Celniker SE, Holt RA, Evans CA, Gocayne JD, Amanatides PG et al. (2000). The genome sequence of Drosophila melanogaster. Science 287: 2185-2195.

Aulard S, Vaudin P, Ladeveze V, Chaminade N, Periquet G, Lemeunier F (2004). Maintenance of a large pericentric inversion generated by the hobo transposable element in a transgenic line of Drosophila melanogaster. Heredity 92: 151-155.
Bazin C, Denis B, Capy P, Bonnivard E, Higuet D (1999). Characterization of permissivity for hobo-mediated gonadal dysgenesis in Drosophila melanogaster. Mol Gen Genet 261: 480-486.

Bellen HJ, Levis RW, Liao G, He Y, Carlson JW, Tsang G et al. (2004). The BDGP gene disruption project: single transposon insertions associated with $40 \%$ of Drosophila genes. Genetics 167: 761-781.

Blackman RK, Grimaila R, Koehler MMD, Gelbart WM (1987). Mobilization of hobo elements residing within the decapentaplegic gene complex: suggestion of a new hybrid dysgenesis in Drosophila melanogaster. Cell 49 497-505.

Harada K, Yukuhiro K, Mukai T (1990). Transposition rates of movable genetic elements in Drosophila melanogaster. Proc Natl Acad Sci USA 87: 3248-3252.

Kaminker JS, Bergman CM, Kronmiller B, Carlson J, Svirskas R, Patel S et al. (2002). The transposable elements of the Drosophila melanogaster euchromatin: a genomics perspective. Genome Biol 3: RESEARCH0084.

Kapitonov VV, Jurka J (2003). Molecular paleontology of transposable elements in the Drosophila melanogaster genome. Proc Natl Acad Sci USA 100: 6569-6574.

Lampe DJ, Grant TE, Robertson HM (1998). Factors affecting transposition of the Himar1 mariner transposon in vitro. Genetics 149: 179-187. 
Maisonhaute C, Ogereau D, Hua-Van A, Capy P (2007). Amplification of the 1731 LTR retrotransposon in Drosophila melanogaster cultured cells: origin of neocopies and impact on the genome. Gene 393: 116-126.

Myers EW, Sutton GG, Delcher AL (2000). A whole-genome assembly of Drosophila. Science 287: 2196-2204.

Nuzhdin SV, Pasyukova EG, Mackay TF (1996). Positive association between copia transposition rate and copy number in Drosophila melanogaster. Proc R Soc London B Biol Sci 263: 823-831.

Rouzic AL, Capy P (2005). The first steps of transposable elements invasion: parasitic strategy vs genetic drift. Genetics 169: 1033-1043.
Shaffer CD, Wuller JM, Elgin SC (1994). Preparation of Drosophila nuclei. Methods Cell Biol 44: 185-189.

Smoller DA, Petrov D, Hartl DL (1991). Characterization of bacteriophage P1 library containing inserts of Drosophila DNA of 75-100 kilobase pairs. Chromosoma 100: 487-494.

Tamkun JW, Deuring R, Scott MP, Kissinger M, Pattatucci AM, Kaufman TC et al. (1992). brahma: a regulator of Drosophila homeotic genes structurally related to the yeast transcriptional activator SNF2/SWI2. Cell 68: 561-572.

Vieira C, Biemont C (1997). Transposition rate of the 412 retrotransposable element is independent of copy number in natural populations of Drosophila simulans. Mol Biol Evol 14: 185-188. 